\section{Polymyalgia rheumatica is mainly a synovitis of the large joints}

The exact cause of polymyalgia rheumatica $(P M R)$ is unknown. Studies of patients with PMR and giant-cell arteritis indicate that PMR could be a vasculitis limited to the subclavian or axillary arteries, or a synovitis or perisynovitis of the shoulders and hips. A new study has now shown that PMR is mainly a synovitis of the large joints.

The study included 35 patients with isolated PMR. Patients received methylprednisolone $12 \mathrm{mg} /$ day, which was tapered and discontinued at 6 months. Fluorodeoxyglucose (FDG) deposition was measured by PET scans before treatment, and 3 and 6 months after treatment if possible. FDG uptake was scored at seven vascular regions, and at the shoulder region, hip region, and the processi spinosi of the vertebrae.

Low vascular involvement was noted in $31 \%$ of patients at baseline. By contrast, $94 \%$ of patients had increased FDG uptake in the shoulder, $89 \%$ in the hip and $51 \%$ in the processi spinosi. PET scans at 3 months showed a decrease in the intensity of FDG uptake at both the vascular and proximal joint regions; PET scans at 6 months were difficult to interpret as disease relapse occurred in $58 \%$ of patients. Relapse was not related to specific FDG-PET findings, and therefore repeat FDG-PET scans for PMR follow-up are not recommended.

Although this study indicates that PMR is a manifestation of a synovitis of the proximal joints, distinction between perisynovitis and synovitis was not possible because of the low resolution of the PET scans.

Original article Blockmans D et al. (2007) Repetitive 18-fluorodeoxyglucose positron emission tomography in isolated polymyalgia rheumatica: a prospective study in 35 patients. Rheumatology (Oxford) 46: 672-677

\section{Potential role of interleukin-6 and chemokines in the pathogenesis of neuropsychiatric SLE}

Patients with systemic lupus erythematosus (SLE) can have a wide array of neuropsychiatric manifestations, but despite the high prevalence of these manifestations, an accurate indicator of central nervous system involvement in patients with SLE has not been identified.
Cytokines and chemokines are associated with neuropsychiatric symptoms, and therefore a study has been carried out to elucidate the levels of inflammatory molecules in the cerebrospinal fluid (CSF) of patients with neuropsychiatric SLE.

The study included 42 patients with neuropsychiatric SLE, 6 patients with SLE and septic meningitis, 16 patients with SLE without neuropsychiatric symptoms, and 25 patients with nonautoimmune diseases without neuropsychiatric manifestations. CSF samples from all patients were analyzed using bead flow cytometry to detect levels of cytokines (IL-2, IL-4, IL-6, IL-10, TNF and IFN $\gamma$ ) and chemokines (CCL2, CCL5, CXCL8, CXCL9, and CXCL10). Thirty patients with neuropsychiatric SLE had a second CSF sample taken and analyzed 6 months after the first.

Patients with neuropsychiatric SLE had high levels of the type 2 T-helper lymphocyte cytokine IL-6 and of chemokines compared with patients with nonautoimmune diseases and patients with SLE without neuropsychiatric symptoms. Analysis of the second CSF sample in patients with neuropsychiatric SLE showed that all previously elevated levels of inflammatory molecules had decreased.

This study shows that IL- 6 and chemokines seem to be accurate indicators of neurologic involvement in patients with SLE, although more details of the pathogenesis of neuropsychiatric SLE remain to be elucidated.

Original article Fragoso-Loyo H et al. (2007) Interleukin-6 and chemokines in the neuropsychiatric manifestations of systemic lupus erythematosus. Arthritis Rheum 56: $1242-1250$

\section{Potential link between depression and vitamin $D$ deficiency in patients with fibromyalgia}

A new study has highlighted the possibility that vitamin $\mathrm{D}$ deficiency is associated with anxiety and depression in patients with fibromyalgia. In an earlier investigation, low levels of vitamin $D$ were found in up to $50 \%$ of patients. Vitamin $D$ deficiency is associated with depression, and therefore the investigators aimed to evaluate vitamin $D$ levels in patients with fibromyalgia, and compare them with assessments of fibromyalgia impact, anxiety and depression. 